\title{
Interisland Spacing and Angle Effects on Magnetic Reversal in Permalloy Arrays
}

\author{
H. Koo, C. Krafft, and R. D. Gomez, Member, IEEE
}

\begin{abstract}
The switching characteristics of submicron permalloy elements were systematically studied as a function of interisland spacing. The result was an increase of the switching field with reduction in spacing, with a maximum value for near zero spacing. However, in overlapped segments, all elements switched simultaneously at lower fields than the maximum at zero spacing. The switching field depends on the number of connected segments as described by the Jacobs-Bean model, whereas the switching of the separated islands is governed mainly by the field emanating from the nearest-neighbor magnetic poles. The reversal behavior of the branched contiguous array was also studied. The angle between the branch axis and the easy axis played a critical role in establishing the switching field. The reversal field of the branched segment reached a minimum at $45^{\circ}$ and a maximum at $90^{\circ}$. A coherent rotation model, which includes the effect of the array geometry, appears to explain the trends.
\end{abstract}

Index Terms-Angle, array, coherent rotation, interisland spacing, permalloy, switching field.

\section{INTRODUCTION}

A $\mathrm{S}$ high-density integrations of magnetic devices are realized, the role of the interaction between small single domain islands becomes a very important concern. In a previous paper, [1], we established that interconnected 35-nm-thick $\mathrm{Ni}_{81} \mathrm{Fe}_{19}$ segments switch at a single field in a process that appears to be governed by parallel rotation, as described by modified Jacobs-Bean chain-of-spheres model [2]. In this study, we focus on the switching characteristics of submicron $\mathrm{Ni}_{81} \mathrm{Fe}_{19}$ segments as a function of interisland spacing. The motivation of the current work is to understand the switching characteristics in the transitional regime between indirect and direct coupling of the islands. We also investigated the reversal behavior of the connected segments as a function of branch bend angle. The magnetization reversal of a straight undulated line occurs coherently in contrast with the complex switching behavior of the bent lines. These experiments show magnetic reversal propagation in bent arrays and how interconnected islands interact with neighbors oriented at specific angles.

Manuscript received February 14, 2002; revised May 22, 2002. This work was supported in part by NSF CAREER Award ECS-9984797 and performed in part at the Cornell Nanofabrication Facility (a member of the National Nanofabrication Users Network), which is supported by the National Science Foundation under Grant ECS-9731293, Cornell University, and industrial affiliates.

H. Koo and R. D. Gomez are with Department of Multi-Electrical and Computer Engineering, University of Maryland, College Park, MD 20742 USA and the Laboratory for Physical Sciences, University of Maryland, College Park, MD 20740 USA (e-mail: hkoo@eng.umd.edu).

C. Krafft is with Laboratory for Physical Sciences, University of Maryland, College Park, MD 20740 USA.

Digital Object Identifier 10.1109/TMAG.2002.801947.

\section{EXPERIMENTAL TECHNiQue}

The arrays were patterned using electron beam lithography, with two different molecular weights of polymethyl methacrylate (PMMA) used as double layer resist. The molecular weight of top layer is $950 \mathrm{k}$, and the bottom layer is $450 \mathrm{k}$. The sensitivity of PMMA is controlled by the molecular weight of PMMA. The top layer acts to mask the bottom layer, which is more sensitive to the electron beam. This method forms a sharp undercut of the bottom layer and makes the lift-off process easier. $\mathrm{Ni}_{81} \mathrm{Fe}_{19}$ thin films were deposited by electron beam evaporator under a base pressure of $1 \times 10^{-6}$ Torr. The arrays were placed in a magnetic force microscope (MFM) with a variable external field, and successive magnetic images of the particles were obtained at specific external field values. The magnetic images were obtained using the interleave mode, where topography was recorded in the first scan, and the magnetic image was obtained on the second pass.

\section{RESULTS AND DISCUSSION}

Four different interisland spacings were studied, namely 80 $\mathrm{nm}, 40 \mathrm{~nm}, \sim 0 \mathrm{~nm}$, and $-20 \mathrm{~nm}$. The minus sign indicates that the adjacent islands are overlapped. The topography and MFM images of representative island arrays are shown in Fig. 1. The lateral size of an island is $300 \times 900 \mathrm{~nm}$, and the thickness is 25 $\mathrm{nm}$. In the MFM images on the right, each element corresponds to a dark and bright contrast pair, which arise from the local variation of the magnetization near the edges. By convention, we assume that the magnetization direction in the interior is from bright to dark.

Fig. 2 shows the switching distribution of 20 element arrays at various spacing distances $S$. These curves indicate the effect of the interaction. The peaks of switching curves are 114, 121, and 132 Oe for $S=80,40$, and $\sim 0 \mathrm{~nm}$, respectively, and a clear trend of increasing switching field with decreasing interspacing is observed. Apparently, when the islands are aligned, the stray fields from the nearest neighbors are parallel to the magnetization of an island and thereby stabilize the magnetic moment. In order to switch the islands, the external field should be large enough to overcome not only the intrinsic coercivity but also interaction field of neighboring elements. The magnitude of stray field can be estimated by using monopolar charge distribution. It varies as $r^{-x}$, where the exponent $x$ varies from 0 to 1 , depending on the exact geometry of the island apex. In our case, we estimate the apex as a conical point with a half angle of $45^{\circ}$, which produces an exponent of -0.5 [3]. The switching field of the interacting island is the sum of the intrinsic switching field 

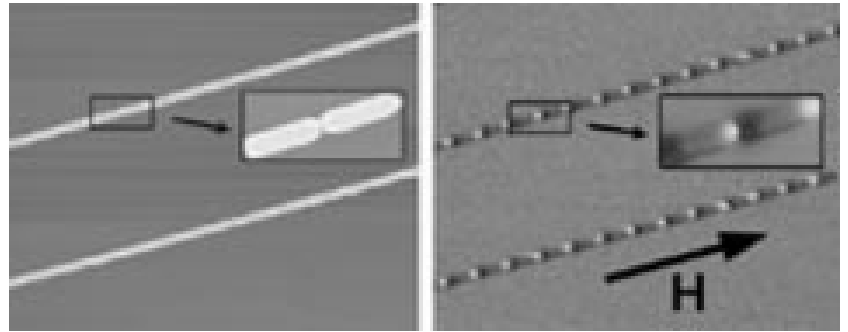

Fig. 1. Topography and MFM image of two segment arrays. Each line consists of 20 segments. The interisland spacing is $40 \mathrm{~nm}$ (upper row) and $80 \mathrm{~nm}$ (lower row).

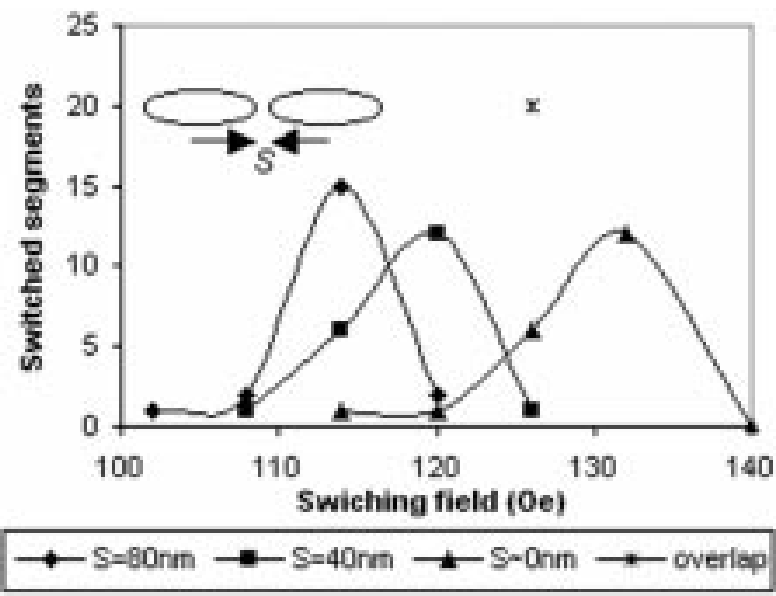

Fig. 2. Switching distribution of 20 element arrays as a function of interspacing.

of an isolated island (96 Oe in average) and the stray field from its neighbors. Using the above argument, we found very good agreement between the $r^{-0.5}$ model and the stray fields, which are 18,25 , and 36 Oe at $80,40,20 \mathrm{~nm}$ spacing, respectively. The stray field is simply calculated from measured switching field. We note that the model fits well if we assume that the closest spacing is $20 \mathrm{~nm}$ as opposed to zero. For $S \sim 0 \mathrm{~nm}$, an atomic force microscope (AFM) image shows near zero spacing. However, the film at the end is very thin, and it could be magnetically dead. The radius of the tip is also about $20 \mathrm{~nm}$. Therefore, the real magnetic charge distance between the islands is larger than seen in the AFM image. The magnetic image also shows that the distance between the poles is $20 \mathrm{~nm}$.

The case of directly coupled or interconnected island arrays has considerably different characteristics. An important behavior of these island arrays is that all segments simultaneously switch at $H=127 \mathrm{Oe}$; therefore, only one point instead of the distribution curve is marked in Fig. 2. The switching field of the connected islands depends on number of segments, which has been previously described [1]. Those results indicate that all connected islands aligned in a straight line switch at the same time because the islands are strongly exchange coupled and produce an interaction field in an identical direction.

A case of considerable interest is when some elements of the array are aligned along a different axis. For this purpose, we fabricated connected island arrays with bends as shown in Fig. 3. In the as-prepared state, the magnetization of each island is uniform and smoothly connects with the neighbors. As
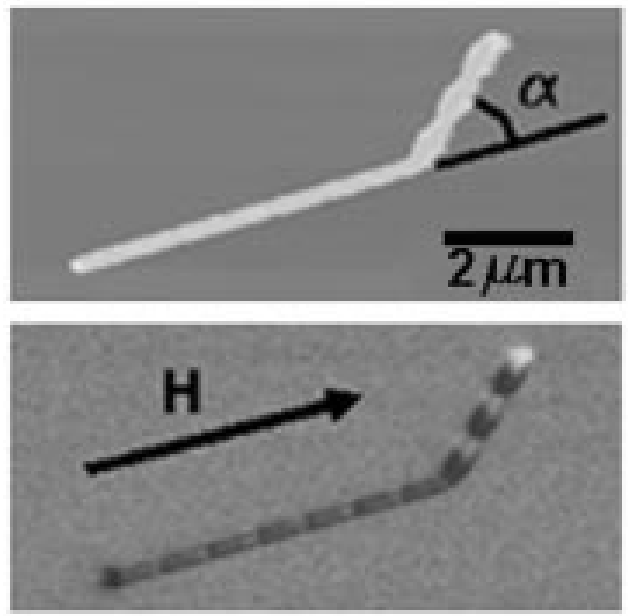

Fig. 3. Topography (top) and MFM image (bottom) of a bent structure. The external field is applied parallel to the main axis.

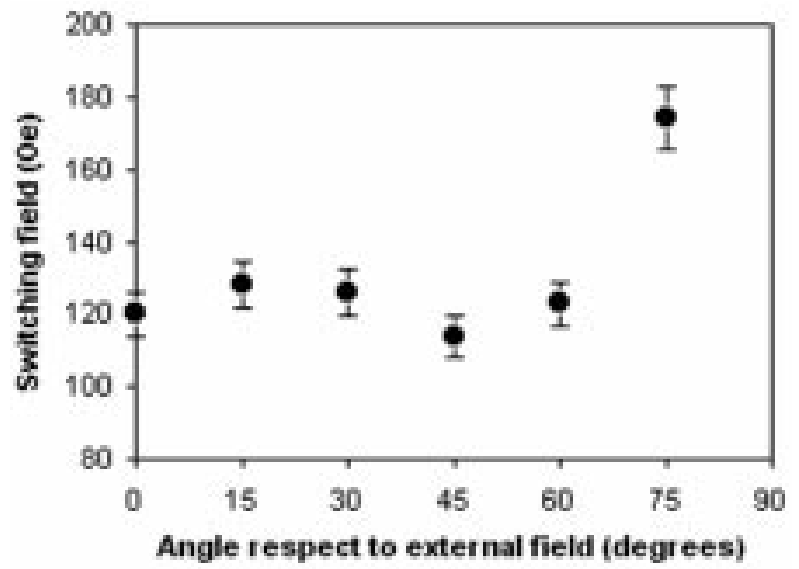

Fig. 4. Switching field of the branch part as a function of angle with respect to the external field.

is evident in the MFM image, positive and negative charges accumulate alternatively, even between the two islands where the bend occurs. In our patterns, the main branch consists of seven elements, and the bent part has three elements. The bent angle with respect to the main axis is denoted by $\alpha$, and the size of a single particle is the same as the previous island. After applying a given field along the main axis, the pattern was imaged at remanence. The main axis element and branch elements have different switching fields, although elements in the same branch switched at the same field. The main part whose element's easy axis is parallel to the field usually switches at lower fields than the bent branch. Moreover, the switching field of the bent branch depended on the angle $\alpha$, as shown in Fig. 4. The reversal field reaches a minimum at $\alpha=45^{\circ}$. When $\alpha$ is near $90^{\circ}$, the reversal field becomes a very sensitive function of the angle, and small increases of the angle cause very large changes in the switching field. At $\alpha=90^{\circ}$, the switching field was in excess of $500 \mathrm{Oe}$.

As before, the switching field can be regarded as the sum of the intrinsic island coercivity and the interaction field with other elements. The coercivity of the single island follows Stoner and Wohlfarth's coherent rotation model [4], reaching the minimum at $45^{\circ}$ and the maximum at $0^{\circ}$ and $90^{\circ}$. In other words the 


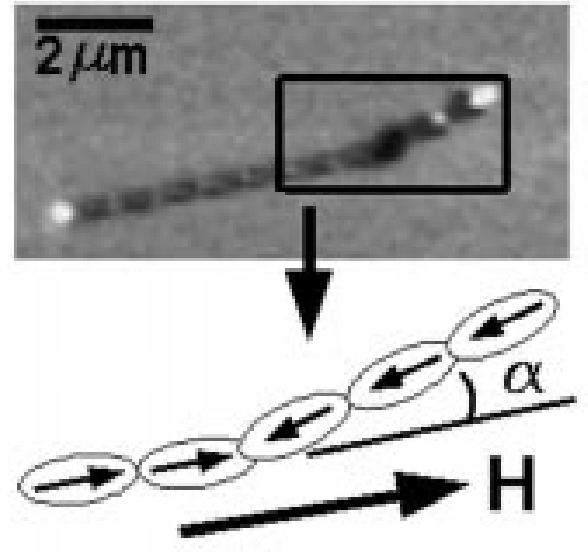

Fig. 5. MFM image and magnetization diagram of a bent structure at an intermediate state. The reversed segments of the main axis reduce the reversal field of the segments of the branch part.

coercivity curve is symmetric about $45^{\circ}$. Seberino and Bertram [5] also reported a similar trend of the magnetic reversal curve using micromagnetic modeling. Their model shows that the switching field slightly decreases with increasing angle up to $45^{\circ}$ and slightly increase again up to near $80^{\circ}$. However, Fig. 4 indicates the coercivity at $75^{\circ}$ is much higher than at $15^{\circ}$. Thus, these models alone cannot explain the observed asymmetry. We believe that the difference is due to the effect of the main axis islands. In the middle of the reversal process, an intermediate state exists. In the intermediate state, the segments in the main parts are already reversed, but the segments of the bent part still retain their initial magnetization. Fig. 5 shows a magnetic image and a magnetization diagram of the intermediate state. At the junction, the magnetization direction of two islands are opposite. The large dark part near the branch junction shows two opposed magnetization vectors in the same region. At both ends, a bright contrast is observed, implying that the same polarity of magnetic charges is accumulated at the edge. This state is relatively unstable in comparison with the state when all elements are aligned in the same direction. At this configuration, the main axis islands, which are already reversed, produce a magnetic field in the same direction as the external field, and it can reduce the switching field. Furthermore, exchange coupling forces neighboring spins to be aligned. The amount of switching field reduction is proportional to $\cos \alpha$. Thus, the effect of the switching field reduction is very low at $\alpha=75^{\circ}$, in contrast with the expectations of the coherent model and the micromagnetic model that the coercivity for $\alpha=15^{\circ}$ and $\alpha=$ $75^{\circ}$ are similar. The observation of Fig. 5 is also noteworthy in that it clearly demonstrates that the magnetization of directly exchange-coupled islands can be oriented opposite each other. This is important in the emerging field of magnetoresistive effects in magnetic constrictions and yields implications for magnetic cellular automata [6].

\section{CONCLUSIONS}

The connected island array has a completely different magnetic behavior from nano-spacing island arrays. In the case of separate island arrays, the interaction is mainly affected by nearest islands, and the stray field is proportional to $r^{-0.5}$. The interaction of neighboring islands reaches the maximum when the spacing is near zero. An important behavior of connected island arrays is that all segments are simultaneously switched and that the switching field depends on the length of the array. We also found that the switching behavior of a bent structure follows a two-step process instead of collective switching. The minimum switching field of the bent branch occurs at an angle of $\alpha=45^{\circ}$. In the middle of the switching process for the exchange-coupled bent structure, two adjacent segments at the junction are at opposite directions from the magnetization. This configuration has not been observed for the straight segment arrays.

\section{ACKNOWLEDGMENT}

The authors appreciate the assistance of Dr. A. Bleier and J. Treichler at the Cornell Nanofabrication Facility for sample preparation.

\section{REFERENCES}

[1] H. Koo, M. Dreyer, V. V. Metlushko, and R. D. Gomez, "Investigation of the magnetic interaction of small permalloy particles," IEEE Trans. Magn., vol. 37, pp. 2049-2051, July 2001.

[2] I. S. Jacobs and C. P. Bean, "An approach to elongated fine-particle magnets," Phys. Rev., vol. 100, pp. 1060-1067, 1955.

[3] J. D. Jackson, Classical Electrodynamics, 3rd ed. New York: Wiley, 1998, pp. 104-107.

[4] E. C. S. Stoner and E. P. Wohlfarth, "A mechanism of magnetic hysterisis in heterogeneous alloys," Phil. Trans. R. Soc. Lond., vol. A240, pp. 599-642, 1948.

[5] C. Seberino and H. N. Bertram, "Numerical study of hysteresis and morphology in elongated tape particles," J. Appl. Phys., vol. 85, pp. $5543-5545,1999$.

[6] R. P. Cowburn and M. E. Welland, "Room temperature magnetic quantum cellular automata," Sci., vol. 287, pp. 1466-1468, 2000. 